\title{
Associação entre os sintomas depressivos e a gordura corporal em pacientes com doença renal crônica em hemodiálise
}

\author{
Association between depressive symptoms \\ and body fat in chronic kidney patients in \\ hemodialysis
}

\author{
Heitor Ribeiro' ${ }^{1}$ \\ Thalita Ferreira ${ }^{2}$ (]) \\ Marvery Duarte 3 (1)
}

\section{Victor Baião4 (1) Antônio Inda-Filho 5 Aparecido Ferreira 6 (1)}

${ }^{1}$ Autor para correspondência. Universidade de Brasília (Brasília), Centro Universitário ICESP (Brasília). Distrito Federal, Brasil. heitorsiqueira95@gmail.com 2,4-6entro Universitário ICESP (Brasília). Distrito Federal, Brasil. thalitalauanna@gmail.com, vmbaiao.v6@gmail.com, indafilho@gmail.com, cidopimentel@gmail.com 3Universidade de Brasília (Brasília). Distrito Federal, Brasil. marveryp@gmail.com

RESUMO | INTRODUÇÃO: O excesso de gordura corporal causa alterações metabólicas complexas que acentuam na patogênese e progressão da doença renal crônica (DRC). Além disto, os aspectos comportamentais negativos, como sintomas depressivos e o sedentarismo, são comuns e podem estar associados ao acúmulo de gordura corporal em pacientes que realizam tratamento em hemodiálise (HD). OBJETIVO: Verificar a associação entre os sintomas depressivos e a gordura corporal em pacientes com DRC em HD. MÉTODO: Trata-se de um estudo transversal de um centro clínico de HD em Brasília-DF.

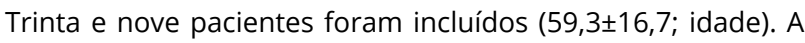
composição corporal foi avaliada por meio de bioimpedância tetrapolar. O inventário de depressão de Beck foi empregado para mensurar os sintomas depressivos. Para analisar a associação entre os sintomas depressivos e a gordura corporal foi adotado o teste de correlação de Spearman. RESULTADOS: Foi observado uma prevalência de depressão de $17,9 \%$. Os sintomas depressivos foram positivamente correlacionados com a gordura corporal $(r=0,42 ; p=0,008)$. CONCLUSÃO: Em nossos resultados, confirmamos que os sintomas depressivos estão associados à gordura corporal em pacientes com DRC em HD. Estes achados demonstram-se importantes para a prática clínica dos profissionais de saúde, sobretudo, nos aspectos dietéticos e psicológicos em pacientes com DRC, de forma que iniciativas de diagnóstico, prevenção e tratamentos sejam priorizadas com o objetivo de reduzir tais condições.

PALAVRAS-CHAVE: Distribuição da Gordura Corporal. Doença Renal Crônica. Depressão. Hemodiálise.

\begin{abstract}
INTRODUCTION: Excess body fat causes complex metabolic changes that enhance the pathogenesis and progression of chronic kidney disease (CKD). Furthermore, negative behavioral aspects such as depressive symptoms and a sedentary lifestyle are common and may be associated with the accumulation of body fat in patients undergoing hemodialysis (HD) treatment. OBJECTIVE: To verify the association between depressive symptoms and body fat in HD patients. METHODS: This is a cross-sectional study that enrolled thirty-nine patients $(59,3 \pm 16,7$; age). The body composition was evaluated by bioimpedance tetrapolar. Beck's depression inventory was used to measure depressive symptoms. Anda, to analyze possible association between depressive symptoms and body fat, Spearman's correlation test was applied. RESULTS: A depression prevalence of $17.9 \%$ was observed. Depressive symptoms were positively correlated with body fat $(r=0.42 ; p=0.008)$. CONCLUSION: Our results confirmed that depressive symptoms were associated with body fat in HD patients. These findings are important for the clinical practice of health professionals, especially in the dietary and psychological aspects; therefore, treatment initiatives for diagnosis, prevention are important to reduce these conditions.
\end{abstract}

KEYWORDS: Body Fat Distribution. Chronic Kidney Disease. Depression. Hemodialysis. 


\section{Introdução}

A doença renal crônica (DRC) é definida como anormalidades na estrutura ou função dos rins, presentes por mais de três meses, com implicações à saúde (KDIGO, 2013). No estágio terminal, o paciente apresenta acúmulo de resíduos, fluidos, desequilíbrio eletrolítico e ácido-base ao ponto de necessitar do tratamento de hemodiálise (HD). Neste momento, o paciente é acometido por uma mudança abrupta de sua rotina em função do tratamento e, somada às complicações advindas desta doença e necessidade do apoio familiar, cria uma barreira à aceitação da nova realidade e condição, o luto, levando à redução da qualidade de vida destes pacientes e o aparecimento de sintomas depressivos (Shirazian, 2019).

A depressão é uma desordem psiquiátrica intimamente associada à qualidade de vida (Debnath et al., 2018). Neste sentido, não somente os aspectos comportamentais, tais como dependência funcional e afastamento das atividades laborais, mas, também, as alterações biológicas e intercorrências clínicas adjacentes à DRC contribuem ao desenvolvimento da depressão. Desta forma, 20\% dos pacientes hemodialíticos experienciam esta desordem, sendo maior do que na população em geral (Ćwiek et al., 2017; McDougall et al., 2018; Shirazian, 2019).

Sabe-se que o excesso de gordura corporal na população em geral vem crescendo nos últimos anos, particularmente em adultos com DRC (Johansen \& Carol, 2017; Ortega et al., 2017). O excesso de gordura corporal resulta em anormalidades metabólicas complexas e abrangentes que aceleram a progressão da DRC (Hall et al., 2014; Hall et al., 2019; Silva et al., 2017). Entre as alterações mais comuns, destacam-se a hiperinflamação, resistência à insulina e a dislipidemia, alterações que dificultam a eficácia do tratamento.

Sendo assim, as consequências do excesso de gordura corporal tornam-se mais um eixo capaz de induzir ao aparecimento de comorbidades e piora do quadro clínico, influenciando negativamente a qualidade de vida e, consequentemente, podendo aumentar a gravidade ou desenvolvimento da depressão. Por outro lado, pacientes depressivos podem apresentar comportamentos nocivos à saúde, como sedentarismo, tabagismo e baixa adesão à dieta, medicamentos e à própria diálise que, por sua vez, impactam na composição corporal (Katon, 2011).

Neste sentido, se faz importante entender que doenças psicossomáticas, como a depressão, desempenham um papel importante (Lee et al., 2016). Contudo, a compreensão sobre a relação entre os sintomas depressivos e a gordura corporal em pacientes com DRC em HD é pouco explorada. Por isso, o objetivo do presente estudo foi verificar a associação dos sintomas depressivos com a gordura corporal em pacientes com DRC em HD. Nossa hipótese é que pacientes com sintomas depressivos apresentariam maior gordura corporal.

\section{Metodologia}

\section{Caracterização do Estudo e Amostra}

Trata-se de um estudo com delineamento transversal, previamente aprovado pelo Comitê de Ética em Pesquisa da Universidade Católica de Brasília $\left(n^{\circ}\right.$ 2.497.191). Os participantes foram recrutados de uma clínica particular localizada em Brasília-DF. Foram incluídos no estudo pacientes em HD que assinaram o Termo de Consentimento Livre e Esclarecido (TCLE), em HD $\geq 3$ meses, liberação médica para a participação do estudo e função cognitiva preservada. Os critérios de exclusão foram: déficit cognitivo que afetou sua participação e incapacidade física de realização das avaliações, tais como amputação de membros e pacientes acamados.

Todos os 74 pacientes foram avaliados de acordo com os critérios de elegibilidade. A Figura 1 apresenta o fluxograma de recrutamento do estudo. 
Figura 1. Fluxograma de recrutamento do estudo

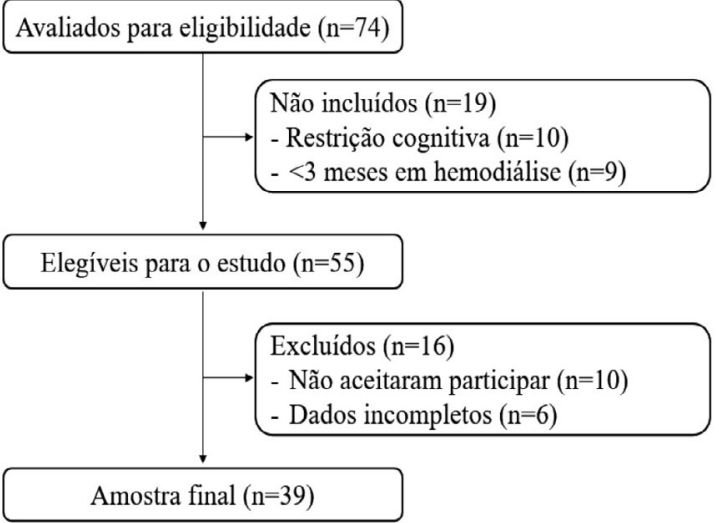

\section{Procedimentos Gerais}

As características sociodemográficas de todos os participantes foram obtidas através dos prontuários médicos. Os participantes foram abordados em dois momentos. No primeiro momento, durante as sessões de HD, foi explicado os objetivos, riscos e benefícios do estudo, posteriormente, assinaram o TCLE. Por último, a aplicação do Inventário de Depressão de Beck (IDB). Em um segundo momento, foram realizadas as avaliações antropométricas e de composição corporal após a sessão de HD realizada no meio de semana. Ademais, o presente estudo respeitou a declaração de Helsinki (1964), bem como as resoluções do Conselho Nacional de Saúde 466/2012.

\section{Inventário de depressão de Beck}

O IDB consiste em 21 itens de múltipla escolha, utilizado para medir a gravidade de sintomas depressivos. O score de cada item varia de acordo com o grau de gravidade do sintoma, sendo classificado 0 a 3 ( 0 = ausência de sintoma, 1 = leve, 2 = moderada e 3 = sintoma grave). A classificação da pontuação final se deu da seguinte maneira: $<10$ = sem depressão ou depressão mínima; de 10-18 = depressão, de leve a moderada; de 19-29 = depressão, de moderada a grave; de 30-63 = depressão grave. Este questionário foi aplicado pelo mesmo avaliador com experiência prévia.

\section{Avaliação antropométrica}

Inicialmente, a massa corporal foi mensurada por meio de uma balança digital portátil (FilizolaTM, Beyond Technology, PL - 200, São Paulo, Brasil), com resolução de 0,1 quilograma (kg). Neste procedimento, foi solicitado aos participantes para estarem com os pés descalços e o peso distribuído igualmente nas duas pernas, ao final, o peso foi registrado em kg. Em seguida, a estatura foi medida por meio de um estadiômetro de parede com resolução de 1 centímetro (FilizolaTM, Beyond Technology, PL - 200, São Paulo, Brasil). Para esta medida, foi empregada a posição anatômica, mantendo-se pés unidos, calcanhares, nádegas e troncos encostados na parede, palmas das mãos voltadas para frente e a cabeça posicionada ao plano de Frankfurt. Através das medidas de massa corporal $(\mathrm{kg})$ e estatura (m), foi possível realizar o cálculo do índice de massa corporal (IMC) por meio da seguinte fórmula: IMC = peso $\div$ estatura2. Todas as avaliações antropométricas foram realizadas em uma sala climatizada, separada dá área de HD, respeitando as recomendações impostas pelos fabricantes e por um avaliador experiente. 


\section{Composição corporal}

A composição corporal foi medida por meio de bioimpedância tetrapolar (Byodinamics, ®310e, São Paulo, Brasil). Para executar o procedimento, foi solicitado aos pacientes que ficassem na posição em decúbito dorsal, assim, foi possível a colocação dos eletrodos nas partes distais do pé e mão direita. Também, solicitado a retirada de qualquer tipo de metal que pudessem comprometer a qualidade das medidas. Para os pacientes que possuíssem marca-passo, a realização do procedimento não foi realizada, respeitando as recomendações do fabricante. As medidas obtidas foram: massa magra (kg), massa gorda (kg), gordura corporal (\%) e taxa metabólica basal (TMB).

\section{Análise Estatística}

Inicialmente, a normalidade dos dados foi verificada por meio do teste de Shapiro-Wilk. Os resultados foram expressos por meio dos valores de média e desvio padrão ( \pm ). Para a comparação das variáveis contínuas entre os grupos, foi utilizado o Teste t de Student para amostras independentes. As associações entre os escores dos sintomas depressivos e a gordura corporal, foi empregada a correlação de Spearman. O valor de significância adotado foi um valor de $p<0,05$. Todas as análises estatísticas foram conduzidas no programa Statistical Package for the Social Sciences (SPSS Inc., Chicago, IL, EUA) versão 22.0 para Windows.

\section{Resultados}

Inicialmente, foram convidados todos os 74 pacientes em HD para participarem. A população elegível foi composta por 55 pacientes, destes, 39 aceitaram participar do estudo. Na tabela 1 são apresentadas as características descritivas e comparações entre o grupo com depressão e sem depressão.

Tabela 1. Caracterização dos pacientes com doença renal crônica em hemodiálise $(n=39)$

\begin{tabular}{|c|c|c|c|c|}
\hline Variáveis & $\begin{array}{l}\text { Todos } \\
(n=39)\end{array}$ & $\begin{array}{l}\text { Com depressão } \\
\qquad(n=7)\end{array}$ & $\begin{array}{l}\text { Sem depressão } \\
\qquad(n=32)\end{array}$ & $\begin{array}{c}\text { Valor de } \\
\text { P }\end{array}$ \\
\hline Idade (anos) & $59,3 \pm 16,7$ & $66,4 \pm 12,7$ & $57,6 \pm 17,2$ & 0,213 \\
\hline Tempo de hemodiálise (meses) & $25,3 \pm 23,8$ & $16,5 \pm 14,5$ & $18,7 \pm 16,0$ & 0,137 \\
\hline \multicolumn{5}{|l|}{ Composição Corporal e Antropometria } \\
\hline Peso (kg) & $70,2 \pm 14,5$ & $68,9 \pm 10,3$ & $70,4 \pm 15,5$ & 0,808 \\
\hline Estatura (m) & $1,64 \pm 0,1$ & $1,60 \pm 0,1$ & $1,65 \pm 0,1$ & 0,175 \\
\hline Índice de Massa Corporal $\left(\mathrm{kg} / \mathrm{m}^{2}\right)$ & $30,9 \pm 6,4$ & $32,5 \pm 6,2$ & $30,5 \pm 6,5$ & 0,479 \\
\hline Circunferência cintura-abdominal (cm) & $99,1 \pm 13,0$ & $100,8 \pm 12,8$ & $98,7 \pm 13,2$ & 0,726 \\
\hline Massa magra $(\mathrm{kg})$ & $46,0 \pm 9,3$ & $42,9 \pm 7,6$ & $46,7 \pm 9,6$ & 0,330 \\
\hline Massa gorda (kg) & $24,6 \pm 9,7$ & $26,3 \pm 7,2$ & $24,2 \pm 10,2$ & 0,609 \\
\hline Gordura corporal (\%) & $34,1 \pm 9,7$ & $37,7 \pm 7,8$ & $33,3 \pm 10,0$ & 0,286 \\
\hline Taxa metabólica basal (kcal) & $1397,2 \pm 286,11$ & $1,303 \pm 232,9$ & $1,418 \pm 295,9$ & 0,343 \\
\hline \multicolumn{5}{|l|}{ Sintomas depressivos } \\
\hline Inventário de depressão de Beck & $6,4 \pm 5,8$ & $16,7 \pm 3,5$ & $3,8 \pm 2,9$ & $<0,001$ \\
\hline
\end{tabular}


A média de idade dos participantes foi de $59,3 \pm 16,7$. O tempo médio de tratamento foi de $25,3 \pm 23,8$ anos e o escore médio do IDB foi $6,4 \pm 5,8$ pontos. Não foram observadas diferenças significativas entre as variáveis sociodemográficas e de composição corporal entre os grupos. As classificações de acordo com os sintomas depressivos obtidos por meio do IDB são apresentadas na Figura 2.

Figura 2. Distribuição das frequências de acordo com as classificações do estado depressivo

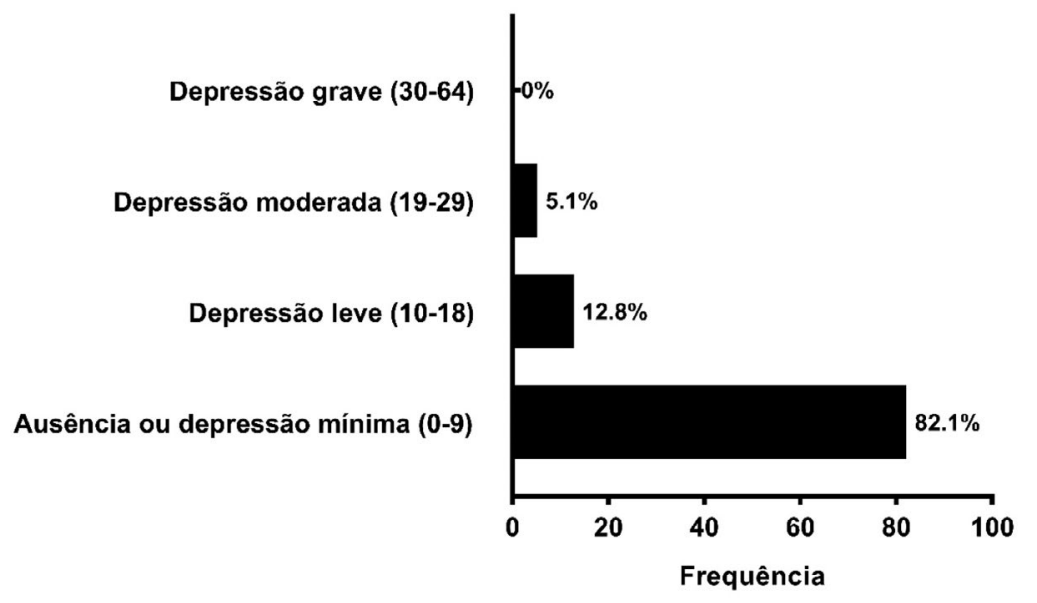

A prevalência de depressão foi de 17,9\%. Não foram identificados pacientes com depressão grave e a sua maioria encontrava-se com ausência ou depressão mínima (82,1\%). A análise de correlação entre os sintomas depressivos e a gordura corporal relativa é apresentada na Figura 3.

Figura 3. Análise de correlação entre os sintomas depressivos e gordura corporal

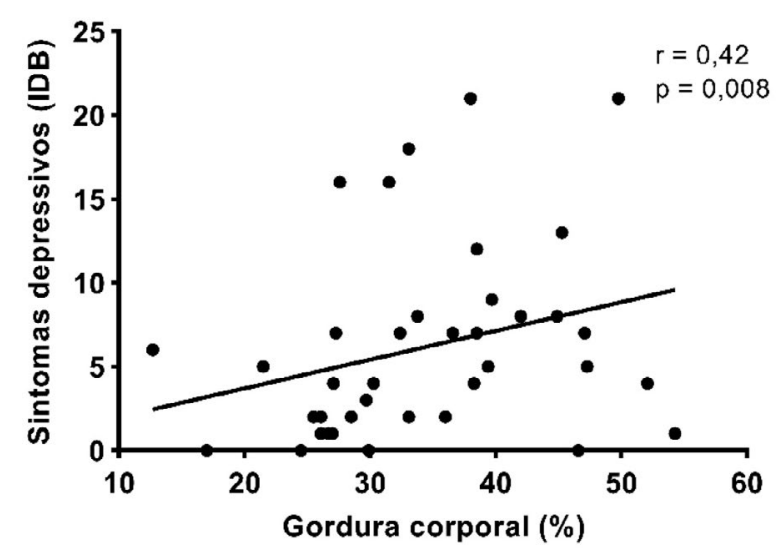

Através da análise de correlação, foi encontrado uma correlação positiva $(r=0,42, p=0,008)$ entre os sintomas depressivos e a gordura corporal relativa na presente amostra. 


\section{Discussão}

O presente estudo avaliou a associação dos sintomas depressivos e a gordura corporal de pacientes com DRC em HD. Os resultados indicam uma correlação moderada entre a gordura corporal e os sintomas depressivos nestes pacientes. A prevalência de depressão encontrada na amostra foi de $17,9 \%$,e a gordura corporal, de $34,1 \pm 9,7 \%$.

A associação entre os sintomas depressivos e as doenças clínicas é frequente, levando a uma pior evolução tanto do quadro psiquiátrico como da doença clínica, com menor aderência às orientações terapêuticas, além de maior morbidade e mortalidade (Jantaratnotai et al., 2017; Milaneschi et al., 2019; Read et al., 2017). Resultados semelhantes aos nossos foram encontrados no estudo de Barros et al. (2016), onde os sintomas depressivos avaliados pelo IDB foram maiores em pacientes obesos em relação aos pacientes com peso dentro da normalidade. Todavia, em nossa análise estratificada, não foi possível observar diferença na gordura corporal entre os pacientes com e sem depressão.

Pacientes com DRC em HD possuem diversas fontes de estresse, como a perda da independência ao realizar as atividades de vida diária, medo, isolamento social, oscilação do apetite, sensação de incerteza em relação ao futuro e diversos outros aspectos relacionados à própria doença. Estes fatores podem desencadear altos níveis de ansiedade, que, por sua vez, possui alta associação com variadas patologias, enquanto na DRC os dois principais fatores que contribuem para o seu surgimento são a cronicidade da doença e seu tratamento rígido com a permanência frequente em um ambiente hospitalar (Valle et al., 2013). Quando, além destas complicações, ocorre também a alteração da imagem corporal, provocada pelo aumento de peso, provoca-se uma desvalorização da autoimagem e diminuição da autoestima. Em consequência disto, diminui-se a sensação de bem-estar e aumenta-se a sensação de inadequação social, podendo desencadear ou agravar sintomas depressivos e de ansiedade nestes pacientes (Heymsfield \& Wadden, 2017). Isto é evidenciado em nossos achados por meio da análise de correlação, onde encontramos uma associação moderada entre os sintomas depressivos e a gordura corporal nos pacientes em HD.
Os nossos achados diferem de um estudo realizado na Inglaterra a partir de questionários com 8,889 indivíduos, que demonstrou que a presença de obesidade e/ou sobrepeso em pacientes com doenças crônicas foi associada a uma diminuição subjetiva do bem-estar físico, porém, não foi associada à deterioração emocional. Por outro lado, os sujeitos com doenças crônicas, sem obesidade associada, apresentavam uma degradação do bem-estar físico e do bem-estar emocional (Doll et al., 2000). Neste sentido, indivíduos com DRC que realizam HD parecem ter uma característica mais específica na associação entre aspectos psicológicos e a quantidade de gordura corporal, quando comparados a indivíduos que possuem outras doenças crônicas.

Vários estudos apontam que o exercício físico pode ser considerado como a melhor opção não-farmacológica na redução dos sintomas depressivos em pacientes ativos ou sedentários com DRC (Wen et al., 2020). O exercício intradialítico mostra-se favorável no declínio dos sintomas depressivos e na redução do peso. Além disso, protocolos periodizados, com momentos distintos entre aquecimento, fase de fortalecimento e resfriamento, foram considerados com uma importante relevância clínica (Ferreira et al., 2021). O uso da restrição calórica em conjunto com a intervenção por exercício físico, também apresentou resultados favoráveis ao controle do peso, redução de percentual de gordura, estresse oxidativo e resposta inflamatória. Na maior parte dos casos, os achados indicam que as intervenções no estilo de vida saudável, de forma isolada ou combinada, retratam uma boa estratégia não-farmacológica para a melhora do estado de saúde mental e física nestes pacientes (Aydemir et al., 2020).

O presente estudo apresenta algumas limitações, dentre elas, o fato de ter sido feito em um único centro, o que limita a extrapolação externa do estudo. Por sua natureza transversal, impede inferências de causalidade. Assim, são necessárias investigações de acompanhamento para estabelecer relações temporais entre os sintomas depressivos e a gordura corporal. Adicionalmente, análises de adipocinas poderiam elucidar melhor a associação destas variáveis com os sintomas depressivos, todavia, possuem um alto custo e baixa factibilidade na rotina clínica. 


\section{Conclusão}

Os resultados observados em nosso estudo demonstram que os sintomas depressivos estão associados à gordura corporal em pacientes com DRC em HD. Neste sentido, intervenções para a melhora dos sintomas depressivos nesta população devem incluir controle dietético, prática de exercício físico intradialítico e estímulos extras para um estilo de vida saudável. Além disso, futuros estudos com desenho intervencional devem ser conduzidos para uma meIhor compreensão do papel da gordura corporal nos sintomas depressivos de pacientes em HD.

\section{Contribuições dos autores}

Ribeiro HS participou do delineamento do estudo, supervisão, coleta de dados, análise dos dados e revisão final. Ferreira $\mathrm{TL}$ participou da supervisão, coleta de dados, análise dos dados e revisão final. Duarte MP e Baião VC participaram da coleta de dados e escrita. Inda-Filho e Ferreira AP participaram do delineamento do estudo, análise dos dados e revisão final.

\section{Conflitos de interesses}

Nenhum conflito financeiro, legal ou político envolvendo terceiros (governo, empresas e fundações privadas, etc.) foi declarado para nenhum aspecto do trabalho submetido (incluindo, mas não se limitando a subvenções e financiamentos, participação em conselho consultivo, desenho de estudo, preparação de manuscrito, análise estatística, etc.).

\section{Referências}

Aydemir, N., Pike, M. M., Alsouqi, A., Headley, S. A. E., Tuttle, K., Evans, E. E., Milch, C. M., Moody, K. A., Germain, M., Lipworth, L., Himmelfarb, J., Ikizler, T. A., \& RobinsonCohen, C. (2020). Effects of Diet and Exercise on Adipocytokine levels in Patients with Moderate to Severe Chronic Kidney Disease [Efeitos da dieta e do exercício sobre os níveis de adipocitocitocina em pacientes com doença renal crônica moderada a grave]. Nutrition, Metabolism and Cardiovascular Diseases. https://doi. org/10.1016/j.numecd.2020.04.012

Barros, A., Costa, B. E., Mottin, C. C., \& D'Avila, D. O. (2016). Depression, quality of life, and body composition in patients with end-stage renal disease: A cohort study [Depressão, qualidade de vida e composição corporal em pacientes com doença renal em fase terminal: um estudo de coorte]. Revista Brasileira de Psiquiatria, 38(4), 301-306. https://doi.org/10.1590/1516-4446-2015-1681
Ćwiek, A., Czok, M., Kurczab, B., Kramarczyk, K., Drzyzga, K., \& Kucia, K. (2017). Association between depression and hemodialysis in patients with chronic kidney disease [Associação entre depressão e hemodiálise em pacientes com doença renal crônica]. Psychiatria Danubina, 29(Suppl 3), 499-503. https://pubmed.ncbi.nlm.nih.gov/28953816/

Debnath, S., O'Connor, J., Hura, C., Kasinath, B., \& Lorenzo, C. (2018). Quality of Life and Depression Among Mexican Americans on Hemodialysis: A Preliminary Report [Qualidade de vida e depressão entre os mexicanoamericanos em hemodiálise: Um Relatório Preliminar]. Therapeutic Apheresis and Dialysis, 22(2), 166-170. https:// doi.org/10.1111/1744-9987.12642

Doll, H. A., Petersen, S. E. K., \& Stewart-Brown, S. L. (2000). Obesity and Physical and Emotional Well-Being: Associations between Body Mass Index, Chronic Illness, and the Physical and Mental Components of the SF36 Questionnaire [Obesidade e Bem-estar Físico e Emocional: Associações entre o Índice de Massa Corporal, Doença Crônica e os Componentes Físicos e Mentais do Questionário SF-36]. Obesity Research, 8(2), 160-170. https://doi.org/10.1038/oby.2000.17

Ferreira, T. L., Ribeiro, H. S., Ribeiro, A. L. A., Bonini-Rocha, A. C., Lucena, J. M. S., Oliveira, P. A., Amorim, F. R. S., Ferreira, A. P., Magno, L. A. V., \& Martins, W. R. (2021). Exercise interventions improve depression and anxiety in chronic kidney disease patients: a systematic review and metaanalysis [Intervenções de exercício melhoram a depressão e a ansiedade em pacientes com doenças renais crônicas: uma revisão sistemática e uma meta-análise]. International Urology and Nephrology, 53(5), 925-933. https://doi. org/10.1007/s11255-020-02612-w

Hall, J. E., Carmo, J. M., Silva, A. A., Wang, Z., \& Hall, M. E. (2019). Obesity, kidney dysfunction and hypertension: mechanistic links [Obesidade, disfunção renal e hipertensão: ligações mecanicistas]. Nature Reviews Nephrology, 15(6), 367-385. https://doi.org/10.1038/ s41581-019-0145-4

Hall, J., Juncos, L., Wang, Z., Hall, M., Carmo, J., \& Silva, A. (2014). Obesity, hypertension, and chronic kidney disease [Obesidade, hipertensão e doença renal crônica]. International Journal of Nephrology and Renovascular Disease, 7, 75. https://doi.org/10.2147/IJNRD.S39739

Heymsfield, S. B., \& Wadden, T. A. (2017). Mechanisms, Pathophysiology, and Management of Obesity [Mecanismos, Fisiopatologia e Gestão da Obesidade]. New England Journal of Medicine, 376(3), 254-266. https://doi. org/10.1056/nejmra1514009

Jantaratnotai, N., Mosikanon, K., Lee, Y., \& McIntyre, R. S. (2017). The interface of depression and obesity [A interface entre depressão e obesidade]. Obesity Research \& Clinical Practice, 11(1), 1-10. https://doi.org/10.1016/j. orcp.2016.07.003 
Johansen, K. L., \& Carol, L. (2017). Body composition in chronic kidney disease Kirsten [Composição corporal na doença renal crônica Kirsten]. Physiology \& Behavior, 176(10), 139-148. https://dx.doi. org/10.1097\%2FMNH.0000000000000120

Katon, W. J. (2011). Epidemiology and treatment of depression in patients with chronic medical illness [Epidemiologia e tratamento da depressão em pacientes com doença médica crônica]. Dialogues in Clinical Neuroscience, 13(1), 7-23. https://doi.org/10.31887/DCNS.2011.13.1/wkaton

KDIGO. (2013). KDIGO 2012 Clinical Practice Guideline for the Evaluation and Management of Chronic Kidney Disease. Kidney International Supplements [KDIGO 2012 Diretrizes de Prática Clínica para a Avaliação e Gerenciamento da Doença Renal Crônica]. Kidney International Supplements, 3(1), 1-150. https://kdigo.org/wp-content/ uploads/2017/02/KDIGO 2012 CKD GL.pdf

Lee, M. C., Wu, S. F. V., Hsieh, N. C., \& Tsai, J. M. (2016). SelfManagement Programs on eGFR, Depression, and Quality of Life among Patients with Chronic Kidney Disease: A Meta-Analysis [Programas de autogestão sobre eGFR, depressão e qualidade de vida entre pacientes com doença renal crônica: Uma Meta-Análise]. Asian Nursing Research, 10(4), 255-262. https://doi.org/10.1016/j. anr.2016.04.002

McDougall, K. A., Larkin, J. W., Wingard, R. L., Jiao, Y., Rosen, S., Ma, L., Usvyat, L. A., \& Maddux, F. W. (2018). Depressive affect in incident hemodialysis patients [Efeito depressivo em pacientes com hemodiálise incidente]. Clinical Kidney Journal, 11(1), 123-129. https://doi.org/10.1093/ckj/sfx054

Milaneschi, Y., Simmons, W. K., van Rossum, E. F. C., \& Penninx, B. W. (2019). Depression and obesity: evidence of shared biological mechanisms [Depressão e obesidade: evidência de mecanismos biológicos compartilhados]. Molecular Psychiatry, 24(1), 18-33. https://doi.org/10.1038/s41380018-0017-5
Ortega, F. B., Lavie, C. J., Bovet, P., Sui, X., \& Chiolero, A. (2017). Trial of Tocilizumab in Giant-Cell Arteritis [Ensaio de Tocilizumab em Giant-Cell Arteritis]. New England Journal of Medicine, 377(15), 1493-1495. https://doi.org/10.1056/ nejmc1711031

Read, J. R., Sharpe, L., Modini, M., \& Dear, B. F. (2017). Multimorbidity and depression: A systematic review and meta-analysis [Multimorbidade e depressão: Uma revisão sistemática e uma meta-análise]. Journal of Affective Disorders, 221, 36-46. https://doi.org/10.1016/j. jad.2017.06.009

Shirazian, S. (2019). Depression in CKD: Understanding the Mechanisms of Disease [Depressão no CKD: Entendendo os Mecanismos da Doença]. Kidney International Reports, 4(2), 189-190. https://doi.org/10.1016/j.ekir.2018.11.013

Silva Junior, G. B., Bentes, A. C. S. N., Daher, E. D. F., \& Matos, S. M. A. (2017). Obesity and kidney disease [Obesidade e doença renal]. Jornal Brasileiro de Nefrologia, 39(1), 65-69. https://doi.org/10.5935/0101-2800.20170011

Valle, L. S., Souza, V. F., \& Ribeiro, A. M. (2013). Estresse e ansiedade em pacientes renais crônicos submetidos à hemodiálise. Estudos de Psicologia, 30(1), 131-138. https:// doi.org/10.1590/s0103-166×2013000100014

Wen, X., Wang, Y., Zhao, Q., Zhang, H., Shi, H., Wang, M., \& Lu, P. (2020). Nonpharmacological Interventions for Depressive Symptoms in End-Stage Renal Disease: A Systematic Review [Intervenções não farmacológicas para sintomas depressivos em doença renal em estágio terminal: uma revisão sistemática]. Western Journal of Nursing Research, 42(6), 462-473. https://doi. org/10.1177/0193945919857540 